\title{
Design of a lunar sample container load bearing frame with transfer function
}

\author{
Haolin Li ${ }^{\mathrm{a}}$, Yixin Liu, Chunyong Wang, Ming Ji and Liang Sun \\ Lanzhou Institute of Physics, Lanzhou, Gansu, China
}

\begin{abstract}
The sample container is one of the key components in the lunar exploration and has to undertake, package and transfer the sample. A new kind of load bearing frame structure is designed to carry the container integrated with interface of the sample transfer. There are three kinds of basic structure in the frame design, $\mathrm{H}$ type, $\mathrm{X}$ type and $*$ type. Through the virtual work principle and Du's article, we calculated their rigidity and compared them. We get the conclusion that the rigidity of $*$ type is better than $\mathrm{X}$ type and $\mathrm{H}$ type. We simulated the recent designed frame with $2 *$ type and $1 \mathrm{X}$ type and get the first six modes, which can also prove that the rigidity of $*$ type is better than $\mathrm{X}$ type. The simulation results prove the fundamental frequency meets the aerospace products requests.
\end{abstract}

Keywords: lunar sample container; load bearing frame; structure.

\section{Guidelines}

The lunar sample container is one of the core components in the Chinese lunar exploration of the third period. Its main function is to undertake and package the lunar sample which to maintain its original state. It also has the transfer function which transfers the sample container to the sample cabin. Before the transfer, the container has to go through complex mechanical environments as launching, transfer from the earth to the moon, landing on the moon and lifting on the moon. During all the environments if the transfer structure rigidity has some changes, it will influence the transfer function, which will influence the success of the sampling task of the detector. A kind of load bearing frame is designed to have the function of undertaking the container and transferring it. In this article, the rationality of the rigidity design of the load bearing frame has been analyzed, and calculated its rigidity to determine the proper structure.

\section{Design of the load bearing frame and analysis}

The load bearing frame mainly provides the installation interface for each component of the sample container including the open/close mechanism. It is made of titanium alloy TC4. The frame is integrated with transfer guide interface and fix interface of the return vehicle. During its machining process, first machine the blank, and do the annealing treatment to reduce the stress in the process and improve the machining precision.

${ }^{a}$ Corresponding author : haolin-lei@163.com

(C) 2016. The authors - Published by Atlantis Press 


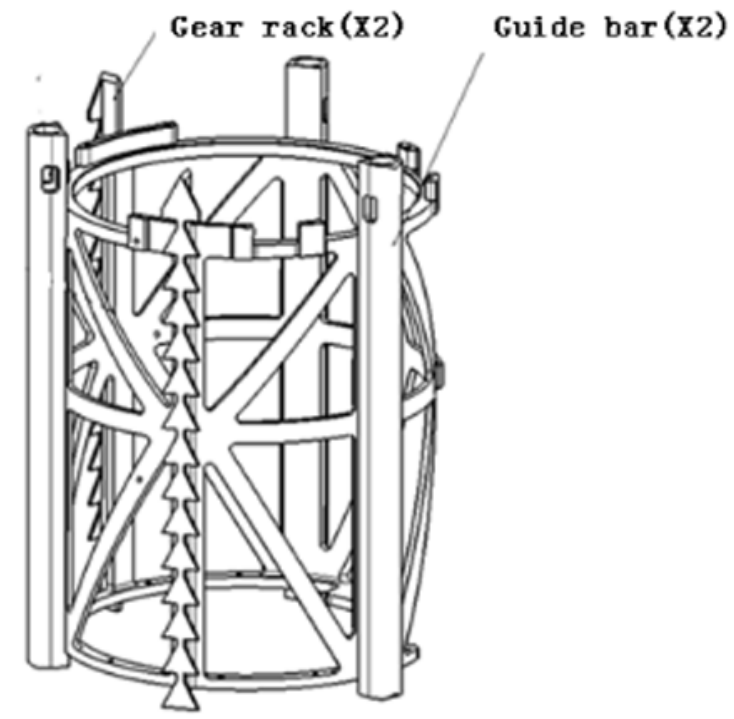

Figure 1. The load bearing frame of the lunar sample container

In the early design there is $\mathrm{H}$ type structure and $\mathrm{X}$ type structure as the basic structure of the frame. In the latest design considering the increased function of the interface with the transfer guide mechanism and the fix interface with the return vehicle, we change the basic structure type as the $*$ type as seen in Fig.1.

\subsection{The rigidity comparison of each basic type}

Simplify each basic type including the $\mathrm{H}$ type, the $\mathrm{X}$ type and the * type, and calculate the rigidity of each type.

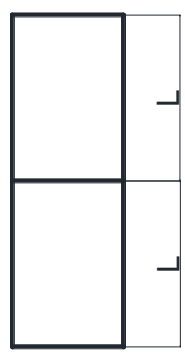

(1)

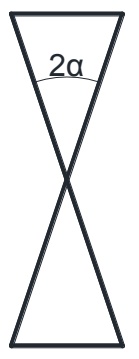

(2)

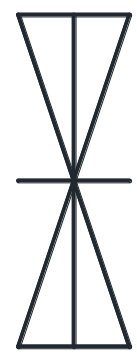

(3)

(1) as the $\mathrm{H}$ type; (2) as the $\mathrm{X}$ type; (3) as the * type

Figure 2. The basic component types

According to Du's article, the transverse rigidity of $\mathrm{H}$ type and $\mathrm{X}$ type have been calculated as following:

$$
\begin{gathered}
K_{1}=\frac{3 E I}{8 L^{3}} \\
K_{2}=\frac{4 E I}{3 L^{3}}+\frac{E A L^{2} \sin ^{2} \alpha}{3 L^{3}}
\end{gathered}
$$


In which, $\mathrm{A}$ is the cross sectional area of rod, $\alpha$ is the angle between the rods, $\mathrm{L}$ is the length of the rod as seen in Fig.2, E is the elastic modulus of the material, I is the inertial moment of the rod.

Now we calculate the transverse rigidity of the $*$ type.
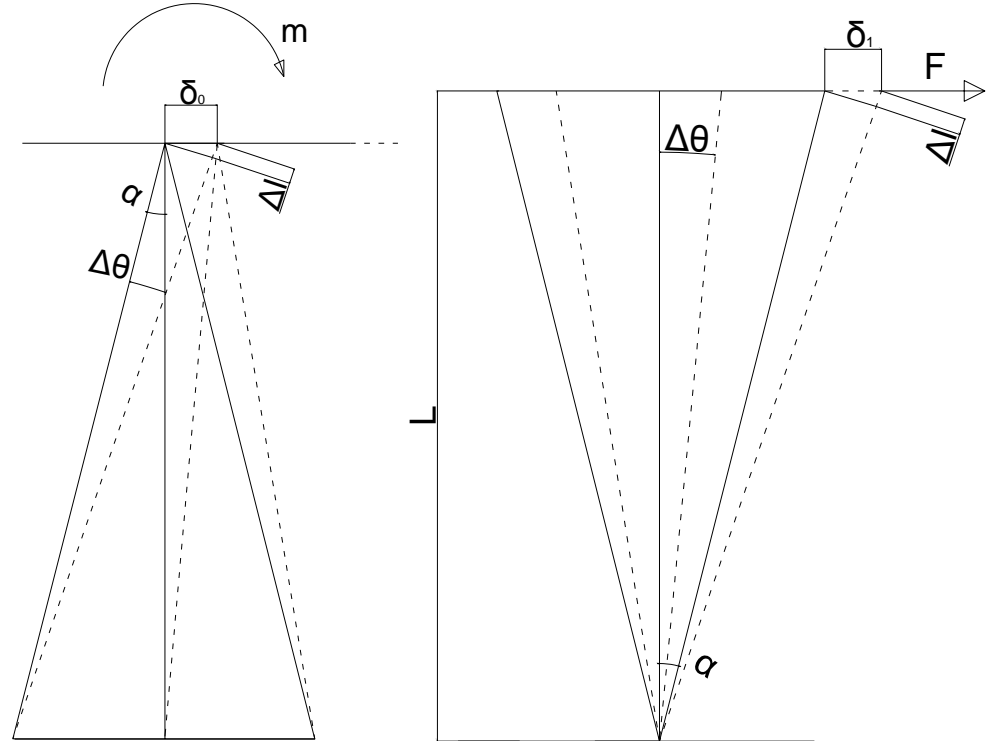

Figure 3. The $*$ type calculation diagram

First calculate the lower part,

$$
\begin{gathered}
m_{1}=m_{2}=m_{3}=\frac{2 E I \delta_{0}}{L^{2}} \\
N_{1}=N_{2}=N_{3}=\frac{E A \delta_{0} \sin \alpha}{L}
\end{gathered}
$$

In which, $\Delta \theta=\frac{\delta_{0}}{L}, \Delta l=\delta_{0} \sin \alpha$.

According to the virtual work principle, we have:

$$
\int N_{1} d(\Delta l)+\int N_{2} d(\Delta l)+\int N_{3} d(\Delta l)+\int N_{4} d(\Delta l)+\int m_{1} d(\Delta \theta)+\int m_{1} d(\Delta \theta)+\int m_{1} d(\Delta \theta)=2 F \delta_{0}
$$

then:

$$
\delta_{0}=\frac{F L^{3}}{E\left(3 I+2 A L^{2} \sin ^{2} \alpha\right)}
$$

In the same way, the upper part is calculated:

$$
\delta_{1}=\frac{F L^{3}}{3 E\left(I+A L^{2} \sin ^{2} \alpha\right)}
$$

Then we have: 


$$
\delta=\delta_{0}+\delta_{1}=\frac{F L^{3}}{E\left(3 I+2 A L^{2} \sin ^{2} \alpha\right)}+\frac{F L^{3}}{3 E\left(I+A L^{2} \sin ^{2} \alpha\right)}
$$

So the rigidity of $*$ type is:

$$
K_{3}=\frac{F}{\delta}=\frac{3 E\left(3 I+2 A L^{2} \sin ^{2} \alpha\right)\left(I+A L^{2} \sin ^{2} \alpha\right)}{L^{3}\left(6 I+5 A L^{2} \sin ^{2} \alpha\right)}
$$

Du's article has proved that $K_{2}>K_{1}$, now we compare $K_{3}$ and $K_{2}$ :

$$
\begin{aligned}
\frac{K_{3}}{K_{2}} & =\frac{9\left(3 I+2 A L^{2} \sin ^{2} \alpha\right)\left(I+A L^{2} \sin ^{2} \alpha\right)}{\left(6 I+5 A L^{2} \sin ^{2} \alpha\right)\left(4 I+A L^{2} \sin ^{2} \alpha\right)} \\
& =\frac{9}{6} \times \frac{3}{4} \times \frac{\left(4 I+\frac{8}{3} A L^{2} \sin ^{2} \alpha\right)}{\left(4 I+A L^{2} \sin ^{2} \alpha\right)} \times \frac{\left(6 I+6 A L^{2} \sin ^{2} \alpha\right)}{\left(6 I+5 A L^{2} \sin ^{2} \alpha\right)}
\end{aligned}
$$

$\frac{K_{3}}{K_{2}}>\frac{27}{24}$, so the rigidity of $*$ type is better than that of $X$ type.

\subsection{Summary}

Actually, the frame of latest design consists $2 *$ type basic structure and $1 \mathrm{X}$ type basic structure, which has better rigidity to ensure the increased function as the transfer guide interface and the fix interface to the return cabin.

\section{Finite element verification}

Analyze the basic frequency of the improved frame. The material of the frame is TC4. Put fixed constraints in the bottom holes. And the The first 6 modal frequencies are shown in Table 1, the vibration modes are shown in Fig.3. The fundamental frequency is $255.23 \mathrm{~Hz}$, which meets the requirement of the aerospace product fundamental as it should be greater than $100 \mathrm{~Hz}$.

Table 1. The first 6 frequencies

\begin{tabular}{lllllll}
\hline Orders & 1 & 2 & 3 & 4 & 5 & 6 \\
\hline $\begin{array}{l}\text { Frequency }( \\
\text { Hz) }\end{array}$ & 255.23 & 256.94 & 359.81 & 367.89 & 443.11 & 652.9 \\
\hline Mode & $\begin{array}{l}\text { Bend } \\
\text { around the } \\
\text { Y axis }\end{array}$ & $\begin{array}{l}\text { Bend around } \\
\text { axis }\end{array}$ & $\begin{array}{l}\text { Bend } \\
\text { around X } \\
\text { and Y axis }\end{array}$ & $\begin{array}{l}\text { Bend } \begin{array}{l}\text { around x } \\
\text { and Y axis }\end{array} \\
\text { axis }\end{array}$ & $\begin{array}{l}\text { Torsion } \\
\text { around } \\
\text { axis Z Z }\end{array}$ & $\begin{array}{l}\text { Bend to Z } \\
\text { axis from 3 } \\
\text { directions }\end{array}$ \\
\hline
\end{tabular}




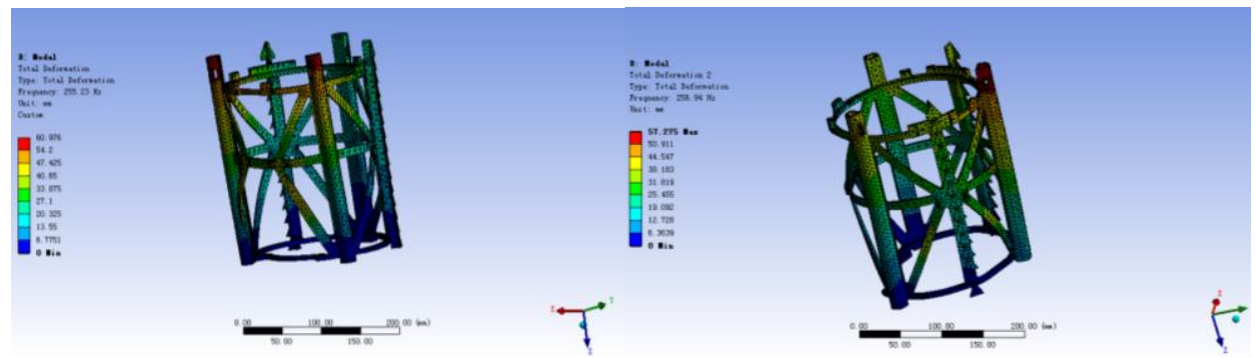

(1)the $1^{\text {st }}$ mode

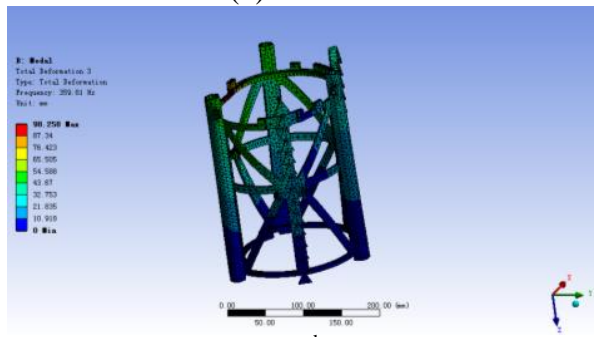

(3)the $3^{\text {rd }}$ mode

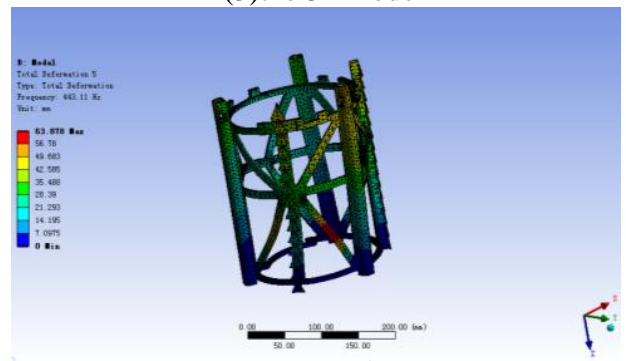

(5)the $5^{\text {th }}$ mode

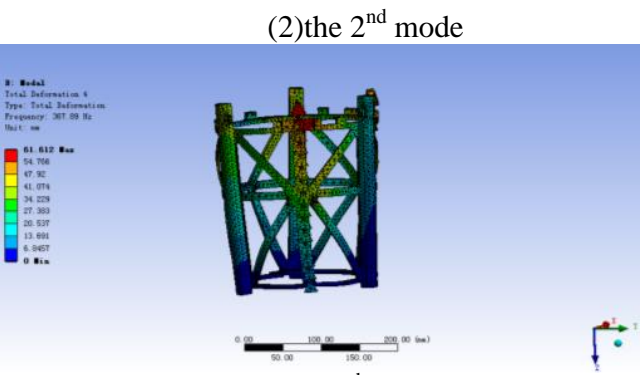

(4)the $4^{\text {th }}$ mode

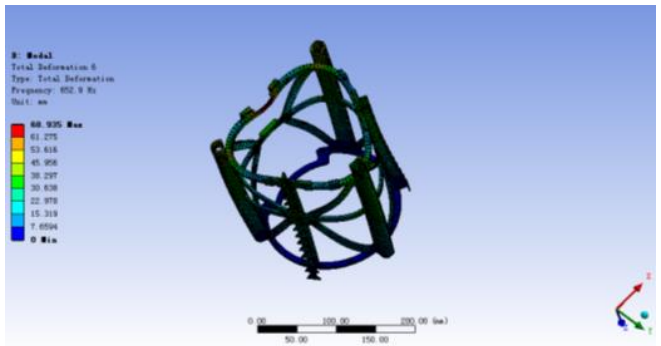

(6)the $6^{\text {th }}$ mode

Figure 4. The finite element analysis of the frame

Actually, the frame of latest design consists of $2 *$ type basic structures and $1 \mathrm{X}$ type basic structure. And from the modes analysis, we can see that the 1st mode happens at the weakness of the $\mathrm{X}$ type structure which is the junction of $\mathrm{X}$, and that can also prove the $*$ type structure is better than $\mathrm{X}$ type structure. On the other hand, the three basic structures are all hyper static structures, which have redundant constraints and distribute the distress evenly. And the rigidity of the hyper static structure is better than that of static structure. So the rigidity of $*$ type structure is better than that of $\mathrm{X}$ structure.

\section{Conclusions}

The load bearing frame is the force suffering structure in the lunar sample container product. During the research and development process, the transfer guide function and the interface function with return cabin are increased, which demand higher requires to the rigidity of the frame. So we change the basic structure of the frame from $\mathrm{X}$ type to * type. The rigidity of different basic structures are calculated through virtual work principle, and the rigidity of * type is better than that of $\mathrm{X}$ type by their comparisons, which is also proved by finite element analysis. And through the finite element analysis, we get the fundamental frequency of the latest designed frame which is more than the request of $100 \mathrm{~Hz}$ in aerospace area. 


\section{References}

1. Du Yonggang, Fu Zhaohui, Yang Zhenchun, etc. The improved design of a lunar sample container's support structure, STRUCTURE \& ENVIRONMENT ENGINEERING,2014,8,41(4)

2. Ralph stone, INC. Design studies and prototype fabrication of lunar sample containers [M]. 1965.

3. J H Allton. Catalog of apollo lunar surface geological sampling tools and containers. Apollo lunar surface journal, 2009.

4. Robert J Erickson. lunar-16-an outstanding new achievement of soviet space science, foreign technology division. 1970, 4.

5. Ye Peijian, Peng Jjing. Deep Space Exploration and Its Prospect in China, ENGINEERING SCIENCE, 2006, 8(10): 13-18.

6. Qu Guangji. Development and Challenge of Spacecraft Dynamics Technology, STRCTURE \& ENVIRONMENT ENGINEERING, 2003, 30(4): 1-6.

7. Liu Hongwen(eds.), Mechanics of materials (Higher Education Press, 1988). 